Draft VERSION OCTOBER 16, 2019

Typeset using LATEX default style in AASTeX61

\title{
PLANCK 2015 CONSTRAINTS ON SPATIALLY-FLAT DYNAMICAL DARK ENERGY MODELS
}

\author{
Junpei Ooba, ${ }^{1, *}$ Bharat Ratra, ${ }^{2}$ And NaOshi Sugiyama ${ }^{1,3,4}$ \\ ${ }^{1}$ Department of Physics and Astrophysics, Nagoya University, Nagoya 464-8602, Japan \\ ${ }^{2}$ Department of Physics, Kansas State University, 116 Cardwell Hall, Manhattan, KS 66506, USA \\ ${ }^{3}$ Kobayashi-Maskawa Institute for the Origin of Particles and the Universe, Nagoya University, Nagoya, 464-8602, Japan \\ ${ }^{4}$ Kavli Institute for the Physics and Mathematics of the Universe (Kavli IPMU), The University of Tokyo, Chiba 277-8582, Japan
}

(Dated: October 16, 2019)

\begin{abstract}
We determine constraints on spatially-flat tilted dynamical dark energy XCDM and $\phi \mathrm{CDM}$ inflation models by analyzing Planck 2015 cosmic microwave background (CMB) anisotropy data and baryon acoustic oscillation (BAO) distance measurements. XCDM is a simple and widely used but physically inconsistent parameterization of dynamical dark energy, while the $\phi \mathrm{CDM}$ model is a physically consistent one in which a scalar field $\phi$ with an inverse powerlaw potential energy density powers the currently accelerating cosmological expansion. Both these models have one additional parameter compared to standard $\Lambda \mathrm{CDM}$ and both better fit the $\mathrm{TT}+$ lowP + lensing $+\mathrm{BAO}$ data than does the standard tilted flat- $\Lambda$ CDM model, with $\Delta \chi^{2}=-1.26(-1.60)$ for the XCDM ( $\phi$ CDM) model relative to the $\Lambda \mathrm{CDM}$ model. While this is a $1.1 \sigma(1.3 \sigma)$ improvement over standard $\Lambda$ CDM and so not significant, dynamical dark energy models cannot be ruled out. In addition, both dynamical dark energy models reduce the tension between the Planck $2015 \mathrm{CMB}$ anisotropy and the weak lensing $\sigma_{8}$ constraints.
\end{abstract}

Keywords: cosmic background radiation — cosmological parameters - large-scale structure of universe - observations

* ooba.jiyunpei@f.mbox.nagoya-u.ac.jp 


\section{INTRODUCTION}

The standard cosmological model, spatially-flat $\Lambda$ CDM (Peebles 1984), is parameterized by six cosmological parameters conventionally taken to be: $\Omega_{\mathrm{b}} h^{2}$ and $\Omega_{\mathrm{c}} h^{2}$, the current values of the baryonic and cold dark matter (CDM) density parameters multiplied by $h^{2}$ [where $h=H_{0} /\left(100 \mathrm{~km} \mathrm{~s}^{-1} \mathrm{Mpc}^{-1}\right)$ and $H_{0}$ is the Hubble constant]; $\theta$, the angular diameter distance as a multiple of the sound horizon at recombination; $\tau$, the reionization optical depth; and $A_{\mathrm{s}}$ and $n_{\mathrm{s}}$, the amplitude and spectral index of the (assumed) power-law primordial scalar energy density inhomogeneity power spectrum (Planck Collaboration 2016). In this model, the currently accelerating cosmological expansion is powered by the cosmological constant $\Lambda$ which is equivalent to a dark energy ideal fluid with equation of state parameter $w_{0}=-1$. For reviews of this model see Ratra \& Vogeley (2008), Martin (2012), and Brax (2018). This model assumes flat spatial hypersurfaces, which is largely consistent with most available observational constraints (Planck Collaboration 2016, and references therein). ${ }^{1}$

However, there also are suggestions that flat- $\Lambda \mathrm{CDM}$ might not be as compatible with different or larger compilations of cosmological measurements (Sahni et al. 2014; Ding et al. 2015; Solà et al. 2015; Zheng et al. 2016 ; Solà et al. 2017a, 2018, 2017b; Zhao et al. 2017; Solà et al. 2017c; Zhang et al. 2017; Solà et al. 2017d; Gómez-Valent \& Solà

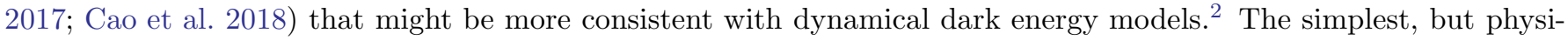
cally inconsistent and widely used, dynamical dark energy parameterization is the seven parameter XCDM model in which the equation of state relating the pressure and energy density of the dark energy fluid is $p_{X}=w_{0} \rho_{X}$ and $w_{0}$ is the additional, seventh, parameter. The simplest physically consistent dynamical dark energy model is the seven parameter $\phi \mathrm{CDM}$ model, in which a scalar field $\phi$ with potential energy density $V(\phi) \propto \phi^{-\alpha}$ is the dynamical dark energy (Peebles \& Ratra 1988; Ratra \& Peebles 1988) and $\alpha>0$ is the seventh parameter that governs dark energy evolution. ${ }^{3}$ In this paper we use the Planck 2015 CMB anisotropy data to constrain the seven parameter spatially-flat XCDM and $\phi$ CDM models. Ooba et al. (2018c) were the first to derive proper (non-approximate) CMB anisotropy data constraints on the physically consistent (non-flat) dynamical dark energy $\phi$ CDM model. ${ }^{4}$ In this paper we present results from the first complete (non-approximate) analyses of CMB anisotropy data using the spatially-flat tilted $\phi \mathrm{CDM}$ model.

The structure of our paper is as follows. In Sec. II we summarize the methods we use in our analyses here. Our parameter constraints are tabulated, plotted, and discussed in Sec. III, where we also comment on the goodness-of-fit of the best-fit XCDM and $\phi$ CDM models. We conclude in Sec. IV.

\section{METHODS}

In the XCDM parameterization the equation of state of the dark energy fluid is $p_{X}=w_{0} \rho_{X}$. In this parameterization, to render it physically sensible, we make the additional (somewhat arbitrary) assumption that spatial inhomogeneities in the dark energy fluid propagate at the speed of light.

In the $\phi \mathrm{CDM}$ model the equations of motion are

$$
\begin{gathered}
\ddot{\phi}+3 \frac{\dot{a}}{a} \dot{\phi}-\kappa \alpha m_{P}^{2} \phi^{-(\alpha+1)}=0 \\
\left(\frac{\dot{a}}{a}\right)^{2}=\frac{8 \pi}{3 m_{P}^{2}}\left(\rho+\rho_{\phi}\right), \\
\rho_{\phi}=\frac{m_{P}^{2}}{32 \pi}\left(\dot{\phi}^{2}+2 \kappa m_{P}^{2} \phi^{-\alpha}\right) .
\end{gathered}
$$

Here the scalar field potential energy density $V(\phi)=\kappa m_{P}^{2} \phi^{-\alpha}, m_{p}$ is the Planck mass, and $\kappa$ is determined in terms of the other parameters. $a$ is the cosmological scale factor and an overdot represents a derivative with respect to time.

\footnotetext{
${ }^{1}$ Using a physically consistent non-flat inflation model (Gott 1982; Hawking 1984; Ratra 1985) power spectrum of energy density inhomogeneities (Ratra \& Peebles 1995; Ratra 2017) to analyse the Planck 2015 cosmic microwave background (CMB) anisotropy measurements (Planck Collaboration 2016), Ooba et al. (2018a) find that these data do not require flat spatial hypersurfaces in the six parameter non-flat $\Lambda$ CDM model (also see Park \& Ratra 2019a,b,d). In the non-flat $\Lambda$ CDM model, compared to the standard flat- $\Lambda$ CDM model, there is no simple tilt option so $n_{\mathrm{s}}$ is no longer a free parameter and it is instead replaced by the current value of the spatial curvature energy density parameter $\Omega_{\mathrm{k}}$. CMB anisotropy data also do not require flat spatial hypersurfaces in the seven parameter non-flat XCDM and $\phi$ CDM inflation models (Ooba et al. 2018b,c; Park \& Ratra 2019b, 2018, 2019d). In both these models $n_{\mathrm{s}}$ is again replaced by $\Omega_{\mathrm{k}}$. These models differ from the seven parameter spatially-flat XCDM and $\phi \mathrm{CDM}$ inflation models we study in this paper, in which $n_{\mathrm{S}}$ is a parameter but $\Omega_{\mathrm{k}}$ is not.

2 Amongst these analyses that also make use of CMB anisotropy data, those that have used a physically consistent dynamical dark energy model such as $\phi$ CDM (Solà et al. 2017b,c,d; Gómez-Valent \& Solà 2017) have performed only an approximate CMB anisotropy analysis.

${ }^{3}$ While XCDM is often used to model dynamical dark energy, it is not a physically consistent model as it cannot describe the evolution of energy density inhomogeneities. Also, XCDM does not accurately model $\phi$ CDM dark energy dynamics (Podariu \& Ratra 2001).

4 Aside from CMB anisotropy measurements, many other observations have been used to constrain the $\phi$ CDM model (see, e.g., Chen \& Ratra 2004; Samushia et al. 2007; Yashar et al. 2009; Samushia \& Ratra 2010; Chen \& Ratra 2011b; Farooq \& Ratra 2013; Pavlov et al. 2014; Avsajanishvili et al. 2015; Farooq et al. 2017; Solà et al. 2017b,c,d; Zhai et al. 2017; Gómez-Valent \& Solà 2017; Avsajanishvili et al. 2017; Ryan et al. 2018, 2019; Park \& Ratra 2019c,d; Khadka \& Ratra 2019).
} 
$\rho$ and $\rho_{\phi}$ are the energy densities excluding the scalar field and that of the scalar field, respectively. The $\phi$ CDM model equations of motion has a time-dependent attractor or tracker solution and so predictions in this model do not depend on initial conditions (Peebles \& Ratra 1988; Ratra \& Peebles 1988; Pavlov et al. 2013). On this solution, the initially subdominant scalar field energy density evolves in a manner to attempt to become the dominant energy density; this mechanism could partially alleviate the fine-tuning associated with the currently accelerating cosmological expansion.

Figure 1 shows the dynamical evolution of the equation of state parameter (the ratio of pressure to energy density) of dark energy in some $\phi \mathrm{CDM}$ and XCDM models and the effects of dynamical dark energy on the CMB temperature anisotropy spectrum.
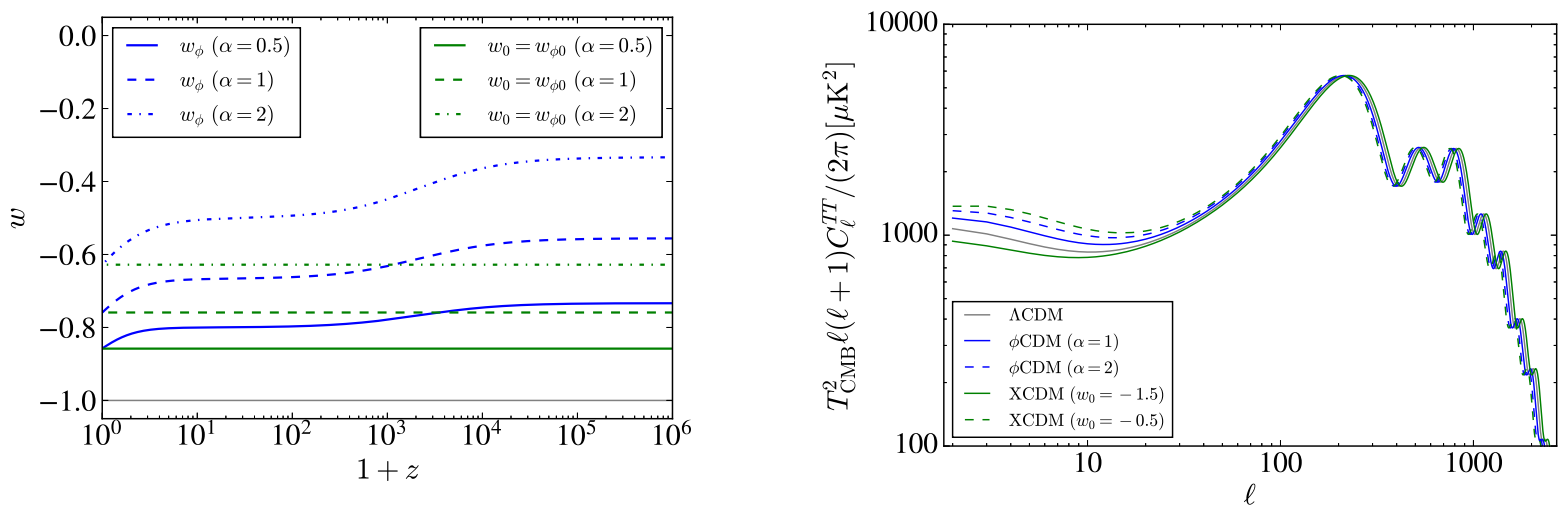

Figure 1. Left panel: Dynamical evolution of the equation of state parameter of dark energy in a few $\phi$ CDM (blue) and corresponding XCDM (green) models. Here $w_{0}$ of each corresponding XCDM model is set to the present value of $w_{\phi}$ of each $\phi \mathrm{CDM}$ model. The $\Lambda \mathrm{CDM}$ model is shown as a gray solid line. Right panel: The CMB temperature anisotropy spectrum of two $\phi$ CDM models (blue lines) and of two XCDM models (green lines). The best-fit $\Lambda$ CDM model is shown as a gray solid line. Here other cosmological parameters are fixed to the best-fit $\Lambda$ CDM model values for the Planck $2015 \mathrm{TT}+$ lowP data.

In this study we compute the angular power spectra of the CMB anisotropy by using CLASS (Blas et al. 2011) ${ }^{5}$ and perform the Markov chain Monte Carlo analyses with Monte Python (Audren et al. 2013). In both spatially-flat dynamical dark energy models the primordial power spectrum of energy density inhomogeneities is taken to be that generated by quantum-mechanical fluctuations in the spatially-flat tilted inflation model (Lucchin \& Matarrese 1985; Ratra 1992, 1989)

$$
P(k)=A_{\mathrm{s}}\left(\frac{k}{k_{0}}\right)^{n_{\mathrm{s}}}
$$

where $k$ is wavenumber and $A_{\mathrm{s}}$ is the amplitude at the pivot scale $k_{0}=0.05 \mathrm{Mpc}^{-1}$.

We consider a flat prior with the ranges of the cosmological parameters chosen to be

$$
\begin{gathered}
100 \theta \in(0.5,10), \quad \Omega_{\mathrm{b}} h^{2} \in(0.005,0.04), \quad \Omega_{\mathrm{c}} h^{2} \in(0.01,0.5), \\
\tau \in(0.005,0.5), \quad \ln \left(10^{10} A_{\mathrm{s}}\right) \in(0.5,10), \quad n_{\mathrm{s}} \in(0.5,1.5),
\end{gathered}
$$

while the parameters characterizing the dark energy dynamics range over

$$
w_{0} \in(-3,0.2), \quad \alpha \in(0,8) .
$$

The CMB temperature and the effective number of neutrinos were set to $T_{\mathrm{CMB}}=2.7255 \mathrm{~K}$ from COBE (Fixsen 2009) and $N_{\text {eff }}=3.046$ with one massive $(0.06 \mathrm{eV})$ and two massless neutrino species in a normal hierarchy. The primordial helium fraction $Y_{\mathrm{He}}$ is inferred from standard Big Bang nucleosynthesis, as a function of the baryon density.

We constrain model parameters by comparing our results to the CMB angular power spectrum data from the Planck 2015 release (Planck Collaboration 2016) and the baryon acoustic oscillation (BAO) distance measurements from the

5 Our flat space $\phi$ CDM CMB anisotropy angular power spectra differ somewhat from earlier results in Brax et al. (2000) and Mukherjee et al. (2003). We have verified that our results are accurate. 
matter power spectra obtained by the 6dF Galaxy Survey (Beutler et al. 2011), the Baryon Oscillation Spectroscopic Survey (LOWZ and CMASS) (Anderson et al. 2014), and the Sloan Digital Sky Survey main galaxy sample (MGS) (Ross et al. 2015).

\section{RESULTS}

In this section we tabulate, plot, and discuss the resulting constraints on the spatially-flat tilted XCDM and $\phi \mathrm{CDM}$ inflation models. Table 1 lists mean values and $68.27 \%$ limits on the cosmological parameters for the XCDM parameterization, and Table 2 lists those for the $\phi \mathrm{CDM}$ model (95.45\% upper limits on $\alpha$ ) ${ }^{6}{ }^{6}$ Figure 2 shows two-dimensional constraint contours and one-dimensional likelihoods from the 4 different $\mathrm{CMB}$ and $\mathrm{BAO}$ data set combinations used in this study. Here all other parameters are marginalized. CMB temperature anisotropy spectra for the best-fit XCDM and $\phi \mathrm{CDM}$ models are shown in Fig. 3, compared to that of the standard spatially-flat tilted $\Lambda$ CDM model. Contours at $68.27 \%$ and $95.45 \%$ confidence level in the $\sigma_{8}-\Omega_{\mathrm{m}}$ plane are shown in Fig. 4, with other parameters marginalized.

Table 1. $68.27 \%$ (95.45\% on an $H_{0}$ ) confidence limits on cosmological parameters of the XCDM parameterization from CMB and BAO data.

\begin{tabular}{lcccc}
\hline \hline Parameter & TT+lowP & TT+lowP+lensing & TT+lowP+BAO & TT+lowP+lensing+BAO \\
\hline$\Omega_{\mathrm{b}} h^{2}$ & $0.02231 \pm 0.00024$ & $0.02229 \pm 0.00024$ & $0.02227 \pm 0.00022$ & $0.02227 \pm 0.00022$ \\
$\Omega_{\mathrm{c}} h^{2}$ & $0.1191 \pm 0.0023$ & $0.1182 \pm 0.0021$ & $0.1189 \pm 0.0019$ & $0.1183 \pm 0.0018$ \\
$100 \theta$ & $1.04195 \pm 0.00046$ & $1.04210 \pm 0.00045$ & $1.04196 \pm 0.00044$ & $1.04205 \pm 0.00042$ \\
$\tau$ & $0.076 \pm 0.020$ & $0.060 \pm 0.018$ & $0.080 \pm 0.020$ & $0.071 \pm 0.017$ \\
$\ln \left(10^{10} A_{\mathrm{s}}\right)$ & $3.085 \pm 0.038$ & $3.049 \pm 0.032$ & $3.092 \pm 0.038$ & $3.071 \pm 0.031$ \\
$n_{\mathrm{s}}$ & $0.9662 \pm 0.0065$ & $0.9678_{-0.0064}^{+0.0060}$ & $0.9669 \pm 0.0058$ & $0.9679 \pm 0.0056$ \\
$w_{0}$ & $-1.95_{-0.61}^{+0.31}$ & $-1.77_{-0.66}^{+0.42}$ & $-1.06 \pm 0.08$ & $-1.03 \pm 0.07$ \\
\hline$H_{0}[\mathrm{~km} / \mathrm{s} / \mathrm{Mpc}]$ & $71.26[2 \sigma$ limit $]$ & $96.08 \pm 21.63$ & $69.35 \pm 1.84$ & $68.91 \pm 1.78$ \\
$\Omega_{\mathrm{m}}$ & $0.150_{-0.069}^{+0.014}$ & $0.172_{-0.092}^{+0.023}$ & $0.294 \pm 0.014$ & $0.296 \pm 0.014$ \\
$\sigma_{8}$ & $1.096_{-0.090}^{+0.17}$ & $1.025_{-0.11}^{+0.19}$ & $0.843 \pm 0.027$ & $0.826 \pm 0.020$ \\
\hline \hline
\end{tabular}

Our Table 1 column 2 and 3 results for XCDM are in good agreement with the Planck 2015 results in Sec. 6.3 of Planck Collaboration (2016) and Tables 21.1 and 21.3 of "Planck 2015 Results: Cosmological Parameter Tables" at wiki.cosmos.esa.int/planckpla2015/images/f/f7/Baseline_params_table_2015_limit68.pdf for most variables (and our Table 1 column 4 and 5 results for XCDM are in good agreement with those of Tables 21.20 and 21.21 of this compilation). However our $w_{0}$ (and derived $H_{0}, \Omega_{\mathrm{m}}$, and $\sigma_{8}$ ) values differ somewhat from the Planck 2015 ones because of the different $H_{0}$ flat prior ranges used (we use $0.2 \leq h \leq 1.3$ here while Planck 2015 used $h \leq 1$ ).

Comparing the Table 1 column $5 \mathrm{TT}+$ lowP + lensing $+\mathrm{BAO}$ results for the spatially-flat tilted XCDM model here to those for the non-flat XCDM model in column 5 of Table 1 in Ooba et al. (2018a), we see that $\Omega_{\mathrm{c}} h^{2}, \Omega_{\mathrm{b}} h^{2}$, $\tau, \ln \left(10^{10} A_{\mathrm{s}}\right), \theta, \Omega_{\mathrm{m}}, w_{0}, H_{0}$, and $\sigma_{8}$ differ by $4.2 \sigma, 2.5 \sigma, 2.2 \sigma, 1.8 \sigma, 1.6 \sigma, 0.66 \sigma, 0.25 \sigma, 0.21 \sigma$, and $0.20 \sigma$ (of the quadrature sum of the two error bars). Similarly for the $\phi \mathrm{CDM}$ case in column 5 of Table 2 here and Table 2 of Ooba et al. (2018c), we find that $\Omega_{\mathrm{c}} h^{2}, \tau, \Omega_{\mathrm{b}} h^{2}, \ln \left(10^{10} A_{\mathrm{s}}\right), \theta, \Omega_{\mathrm{m}}, H_{0}$, and $\sigma_{8}$ differ by $4.2 \sigma, 2.4 \sigma, 2.1 \sigma, 2.0 \sigma, 1.4 \sigma$, $1.3 \sigma, 0.17 \sigma$, and $0.16 \sigma$ (of the quadrature sum of the two error bars). On the other hand, comparing the spatially-flat tilted XCDM and $\phi \mathrm{CDM}$ TT + lowP + lensing $+\mathrm{BAO}$ results we have derived here (and listed in columns 5 of Tables 1 and 2) we see that $H_{0}, \sigma_{8}, \Omega_{\mathrm{m}}, \Omega_{\mathrm{c}} h^{2}, \ln \left(10^{10} A_{\mathrm{s}}\right), n_{\mathrm{s}}, \tau, \Omega_{\mathrm{b}} h^{2}$, and $\theta$ differ by $0.79 \sigma, 0.73 \sigma, 0.66 \sigma, 0.61 \sigma, 0.50 \sigma$, $0.49 \sigma, 0.49 \sigma, 0.36 \sigma$, and $0.24 \sigma$ (of the quadrature sum of the two error bars). We note however that XCDM is not a physical model and so it might not be very meaningful to compare cosmological parameter values measured using the XCDM parameterization and the $\phi \mathrm{CDM}$ model.

In agreement with Park \& Ratra (2019a), who compared cosmological parameter measurements made from cosmological observations by using the spatially-flat tilted $\Lambda \mathrm{CDM}$ model and the non-flat $\Lambda \mathrm{CDM}$ model, we also find that

\footnotetext{
${ }^{6}$ We thank C.-G. Park for pointing out a numerical error in our initial CMB only $\phi$ CDM analyses. Our corrected results here are in very good agreement with those of Park \& Ratra (2018).
} 
when space curvature is allowed to vary many cosmological parameters cannot be determined in a model independent way from cosmological data, with the possible exceptions of $\sigma_{8}$ and $H_{0}$ (and $w_{0}$ in the XCDM parameterization). We emphasize that the somewhat widely held belief that the baryonic matter density $\Omega_{\mathrm{b}} h^{2}$ can be pinned down in a model independent manner by CMB anisotropy and other cosmological observations is not true. ${ }^{7}$ When spatial curvature vanishes it appears that cosmological parameters can be determined in a more model independent fashion, although, again, this is based on using the somewhat arbitrary XCDM parameterization. It is interesting that in this case $H_{0}$ and $\sigma_{8}$ are the most model dependent parameters.

Table 2. $68.27 \%$ (95.45\% on $\alpha$ ) confidence limits on cosmological parameters of the $\phi$ CDM model from CMB and BAO data.

\begin{tabular}{lcccc}
\hline \hline Parameter & TT+lowP & TT+lowP+lensing & TT+lowP+BAO & TT+lowP+lensing+BAO \\
\hline$\Omega_{\mathrm{b}} h^{2}$ & $0.02218 \pm 0.00024$ & $0.02220 \pm 0.00024$ & $0.02239 \pm 0.00021$ & $0.02238 \pm 0.00021$ \\
$\Omega_{\mathrm{c}} h^{2}$ & $0.1199 \pm 0.0023$ & $0.1192 \pm 0.0021$ & $0.1171 \pm 0.0015$ & $0.1169 \pm 0.0014$ \\
$100 \theta$ & $1.04184 \pm 0.00045$ & $1.04193 \pm 0.00044$ & $1.04215 \pm 0.00042$ & $1.04219 \pm 0.00041$ \\
$\tau$ & $0.077 \pm 0.019$ & $0.073 \pm 0.017$ & $0.088 \pm 0.019$ & $0.082 \pm 0.015$ \\
$\ln \left(10^{10} A_{\mathrm{s}}\right)$ & $3.089 \pm 0.037$ & $3.078 \pm 0.030$ & $3.104 \pm 0.037$ & $3.092 \pm 0.028$ \\
$n_{\mathrm{s}}$ & $0.9643 \pm 0.0064$ & $0.9657 \pm 0.0060$ & $0.9714 \pm 0.0051$ & $0.9716 \pm 0.0050$ \\
$\alpha[2 \sigma \mathrm{limit}]$ & $<1.46$ & $<1.19$ & $<0.28$ & $<0.28$ \\
\hline$H_{0}[\mathrm{~km} / \mathrm{s} / \mathrm{Mpc}]$ & $63.37 \pm 3.00$ & $63.69 \pm 3.11$ & $67.32 \pm 0.89$ & $67.33 \pm 0.90$ \\
$\Omega_{\mathrm{m}}$ & $0.357 \pm 0.035$ & $0.352 \pm 0.036$ & $0.308 \pm 0.009$ & $0.307 \pm 0.009$ \\
$\sigma_{8}$ & $0.789 \pm 0.031$ & $0.783 \pm 0.028$ & $0.815 \pm 0.018$ & $0.809 \pm 0.012$ \\
\hline \hline
\end{tabular}

Focusing again on the TT + lowP + lensing + BAO data, we measure $H_{0}=68.91 \pm 1.78(67.33 \pm 0.90) \mathrm{km} \mathrm{s}^{-1} \mathrm{Mpc}^{-1}$ for XCDM $(\phi \mathrm{CDM})$, both of which are consistent with the most recent median statistics estimate of $H_{0}=68 \pm 2.8 \mathrm{~km}$ $\mathrm{s}^{-1} \mathrm{Mpc}^{-1}$ (Chen \& Ratra 2011a), They also are consistent with many other recent estimates (Calabrese et al. 2012; Sievers et al. 2013; Aubourg et al. 2015; Chen et al. 2017; Lin \& Ishak 2017; DES Collaboration 2017b; Yu et al. 2018; Haridasu et al. 2018), although both are lower than the recent local expansion rate determination of $H_{0}=73.45 \pm 1.66$ $\mathrm{km} \mathrm{s}^{-1} \mathrm{Mpc}^{-1}$ (Riess et al. 2018).

The $\mathrm{TT}+$ lowP + lensing $+\mathrm{BAO}$ values of $\tau=0.071 \pm 0.017(0.082 \pm 0.015)$ for XCDM $(\phi \mathrm{CDM})$ measured here are a bit larger than the value of $\tau=0.066 \pm 0.013$ measured using TT + lowP + lensing + New BAO data in the tilted flat- $\Lambda$ CDM model (Park \& Ratra 2019a), but not as large as the values of $\tau$ found in the non-flat models, for TT + lowP + lensing + NewBAO in non-flat $\Lambda \mathrm{CDM} \tau=0.115 \pm 0.011$ (Park \& Ratra 2019a), and for TT + lowP + lensing $+\mathrm{BAO}$ in non-flat XCDM $(\phi \mathrm{CDM}) \tau=0.121 \pm 0.015(0.129 \pm 0.013)$ (Ooba et al. 2018b,c, Tables 1). The larger value for $\tau$ in the non-flat $\Lambda$ CDM case has very interesting implications for reionization (Mitra et al. 2018, 2019).

In both dynamical dark energy models, XCDM and $\phi \mathrm{CDM}$, the data favor non-evolving dark energy, although they are not yet good enough to rule out the possibility of mild dark energy time evolution. More and better-quality data will be needed to resolve this issue. The situation in the non-flat models is quite different, where the data favor mildly closed models in which the curvature energy density contributes about a per cent to the current cosmological energy budget (Ooba et al. 2018a,b,c; Park \& Ratra 2019a,b, 2018, 2019d), at 5.2 $\sigma$ significance in the non-flat $\Lambda$ CDM case for the biggest compilation of reliable cosmological observations (Park \& Ratra 2019b).

The Dark Energy Survey (DES Collaboration 2017a) measures $\Omega_{\mathrm{m}}=0.264_{-0.019}^{+0.032}$ and $\sigma_{8}=0.807_{-0.041}^{+0.062}$ (DES Y1 All, both $68.27 \%$ confidence limits). Our XCDM and $\phi \mathrm{CDM}$ TT + lowP + lensing + BAO results are consistent with these limits (with our $\phi \mathrm{CDM} \Omega_{\mathrm{m}}$ value being the most deviant, high by $1.3 \sigma$ of the quadrature sum of the two error bars). The Dark Energy Survey constraints are also consistent with the XCDM and $\phi$ CDM confidence level contours in the $\sigma_{8}-\Omega_{\mathrm{m}}$ plane shown in Fig. 4 , but are a little more difficult to reconcile with the standard tilted flat- $\Lambda$ CDM model results. Gómez-Valent \& Solà (2017) draw a similar conclusion for these models. The non-flat models are also

${ }^{7}$ See Penton et al. (2018) for a discussion of how observed deuterium abundances can be used to constrain spatial curvature. 

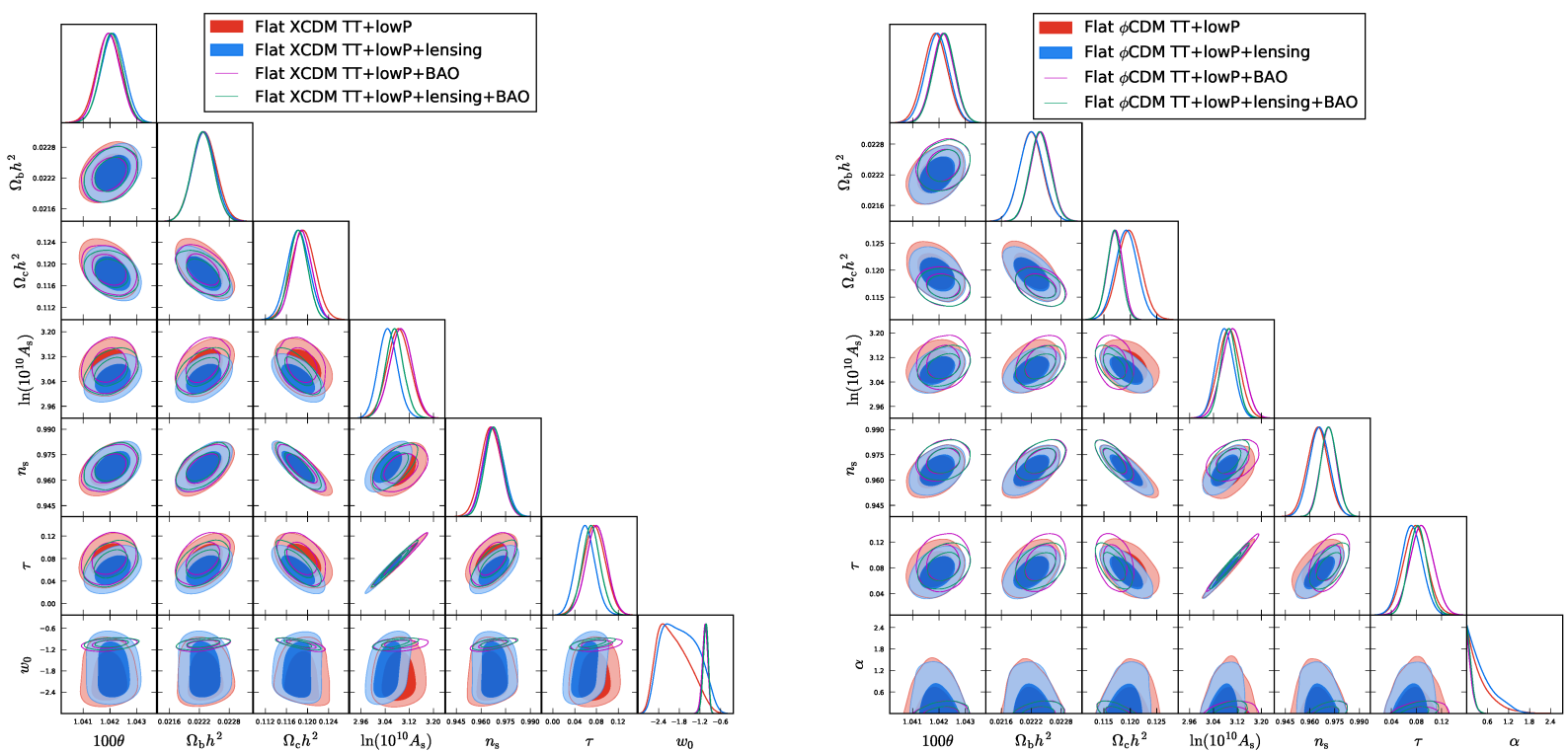

Figure 2. $68.27 \%$ and $95.45 \%$ confidence level contours for the XCDM and $\phi$ CDM models using various data sets, with the other parameters marginalized.

more consistent with the weak lensing constraints (Ooba et al. 2018a,b,c; Park \& Ratra 2019a,b, 2018, 2019d) than is the standard $\Lambda$ CDM model.

As can be seen from Fig. 3 here, and the corresponding ones for the non-flat models (Ooba et al. 2018a,b,c; Park \& Ratra 2019a,b, 2018), the spatially-flat tilted XCDM and $\phi$ CDM models do not do as well at fitting the lower- $\ell C_{\ell}$ temperature data as do the non-flat models, but the flat models better fit the higher- $\ell C_{\ell}$ 's than do the non-flat ones.

While both spatially-flat dynamical dark energy models considered here are more consistent with the weak lensing constraints than is tilted flat- $\Lambda \mathrm{CDM}$, the XCDM parameterization and the $\phi \mathrm{CDM}$ model both have one extra parameter so it is necessary to quantify how well these models fit the totality of data. Table 3 shows $\Delta \chi_{\text {eff }}^{2}$ values for the spatially-flat XCDM and $\phi \mathrm{CDM}$ models relative to the flat- $\Lambda \mathrm{CDM}$ model. Here $\chi_{\mathrm{eff}}^{2}$ is determined from the maximum value of the likelihood, $\chi_{\text {eff }}^{2}=-2 \ln \left(\mathrm{L}_{\max }\right)$. Unlike the non-flat $\Lambda \mathrm{CDM}, \mathrm{XCDM}$, and $\phi \mathrm{CDM}$ models which are not straightforwardly related to the standard $\Lambda$ CDM model (Ooba et al. 2018a,b,c; Park \& Ratra 2019a,b, 2018), the tilted spatially-flat XCDM and $\phi \mathrm{CDM}$ models here are single parameter extensions of the tilted flat- $\Lambda \mathrm{CDM}$ model and so we are comparing nested models here. In this case we can work around the ambiguity in the number of Planck 2015 data points and translate the $\Delta \chi_{\text {eff }}^{2}$ values of Table 3 to relative probabilities. From Table 3 , for the TT + lowP + lensing $+\mathrm{BAO}$ case, the XCDM parameterization and the $\phi \mathrm{CDM}$ model, from $\sqrt{-\Delta \chi_{\mathrm{eff}}^{2}}$ for one additional free parameter, are $1.1 \sigma$ and $1.3 \sigma$ better fits to the data than is tilted flat- $\Lambda$ CDM. ${ }^{8}$ The corresponding $p$ values are 0.26 and 0.21 with one additional degree of freedom. These results indicate that the improvement in fit, in going from tilted flat- $\Lambda \mathrm{CDM}$ to one of the tilted spatially-flat dynamical dark energy models, is not significant. On the other hand, the dynamical dark energy models cannot be ruled out and continue to be of interest, especially $\phi \mathrm{CDM}$ which is a physically consistent model. This means that more and better-quality data will be needed to determine whether the dark energy density is constant or decreases slowly with time. For goodness-of-fit, one may also consider the AIC, which will penalize the dynamical dark energy models for the additional parameter. From $\Delta$ AIC the XCDM $(\phi \mathrm{CDM})$ model is only $69 \%(82 \%)$ as likely as the standard tilted flat- $\Lambda \mathrm{CDM}$ model, again not a strong result either way.

\section{CONCLUSION}

We present constraints on the tilted spatially-flat XCDM and $\phi \mathrm{CDM}$ inflation models determined by analyzing Planck 2015 CMB anisotropy data as well as BAO distance measurements. XCDM is a simply parameterized dynamical dark energy model, and $\phi \mathrm{CDM}$ is a physically consistent one in which a scalar field $\phi$ with an inverse power-law potential

8 Closed- $\phi$ CDM is also the best fitting of the three closed models when BAO data is included (Ooba et al. 2018c). 
Table 3. $\Delta \chi_{\text {eff }}^{2}$ values for the best-fit XCDM ( $\phi$ CDM) model.

\begin{tabular}{lc}
\hline \hline Data sets & $\Delta \chi_{\text {eff }}^{2}$ \\
\hline TT+lowP & $-3.27(+0.41)$ \\
TT+lowP+lensing & $-1.89(-0.79)$ \\
TT+lowP+BAO & $-0.82(-0.64)$ \\
TT+lowP+lensing+BAO & $-1.26(-1.60)$ \\
\hline \hline
\end{tabular}

energy density acts as dynamical dark energy and powers the currently accelerating cosmological expansion. Both of these dynamical dark energy models better fit, although not significantly so, the $\mathrm{TT}+$ low + lensing $+\mathrm{BAO}$ data combination than does the tilted flat- $\Lambda \mathrm{CDM}$ model. Perhaps more interestingly, the dynamical dark energy models reduce the tension between the Planck $2015 \mathrm{CMB}$ anisotropy and the weak lensing $\sigma_{8}$ constraints. More and better data, which should soon be available, is needed to determine if dynamical dark energy can be ruled out, or if dark energy is dynamical.

\section{ACKNOWLEDGMENTS}

We thank G. Horton-Smith and C.-G. Park for helpful discussions. We thank the referee for comments that helped us improve the paper. This work is supported by Grants-in-Aid for Scientific Research from JSPS (Nos. 16J05446 (J.O.) and 15H05890 (N.S.)). B.R. is supported in part by DOE grant DE-SC0019038.

\section{REFERENCES}

Anderson, L., Aubourg, É., Bailey, S., et al. 2014, MNRAS, 441, 24 [arXiv:1312.4877]

Aubourg, É., Bailey, S., Bautista, J. E., et al. 2015, PhRvD, 92, 123516 [arXiv:1411.1074]

Audren, B,, Lesgourgues, J., Benabed, K., \& Prunet, S. 2013, JCAP, 1302, 001 [arXiv:1210.7183]

Avsajanishvili, O., Huang, Y., Samushia, L., \& Kahniashvili, T. 2017, arXiv:1711.11465

Avsajanishvili, O., Samushia, L., Arkhipova, N. A., \& Kahniashvili, T. 2015, arXiv:1511.09317

Beutler, F., Blake, C., Colless, M., et al. 2011, MNRAS, 416, 3017 [arXiv:1106.3366]

Blas, D, Lesgourgues, J., \& Tram, T. 2011, JCAP, 1107, 034 [arXiv:1104.2933]

Brax, P. 2018, Rep. Prog. Phys., 81, 016902

Brax, P, Martin, J., \& Riazuelo, A. 2000, PhRvD, 62, 103505 [arXiv:astro-ph/0005428]

Calabrese, E., Archidiacono, M., Melchiorri, A., \& Ratra, B. 2012, PhRvD, 86, 043520 [arXiv:1205.6753]

Cao, S.-L., Duan, X.-W., Meng, X.-L.,\& Zhang, T.-J. 2018, Eur. Phys. J. C78, 313 [arXiv:1712.01703]

Chen, G., \& Ratra, B. 2004, ApJ, 612, L1

[arXiv:astro-ph/0405636]

Chen, G., \& Ratra, B. 2011a, PASP, 123, 1127 [arXiv:1105.5206]

Chen, Y., Kumar, S., \& Ratra, B. 2017, ApJ, 835, 86 [arXiv:1606.07316]
Chen, Y., \& Ratra, B. 2011b, Phys. Lett. B, 703, 406 [arXiv:1106.4294]

DES Collaboration, Abbott, T. M. C., Abdalla, F. B., Alarcon, A., et al. 2017a, arXiv:1708.01530

DES Collaboration, Abbott, T. M. C., Abdalla, F. B., Annis, J., et al. 2017b, arXiv:1711.00403

Ding, X., Biesiada, M., Cao, S., Li, Z., \& Zhu, Z.-H. 2015, ApJ, 803, L22 [arXiv:1503.04923]

Farooq, O., Madiyar, F. R., Crandall, S., \& Ratra, B. 2017, ApJ, 835, 26 [arXiv:1607.03537]

Farooq, O., \& Ratra, B. 2013, ApJ, 766, L7 [arXiv:1301.5243]

Fixsen, D. J. 2009, ApJ, 707, 916 [arXiv:0911.1955]

Gómez-Valent, A., \& Solà, J. 2017, Europhys. Lett., 120, 39001 [arXiv:1711.00692]

Gott, J. R. 1982, Nature, 295, 304

Haridasu, B. S., Luković, V. V., \& Vittorio, N. 2018, JCAP, 1805, 033 [arXiv:1711.03929]

Hawking, S. W. 1984, Nucl. Phys. B, 239, 257

Khadka, N., \& Ratra, B. 2019, arXiv:1909.01400

Lin, W., \& Ishak, M. 2017, PhRvD, 96, 083532 [arXiv:1708.09813]

Lucchin, F., \& Matarrese, S. 1985, PhRvD, 32, 1316

Martin, J. 2012, C. R. Physique, 13, 566 [arXiv:1205.3365]

Mitra, S., Choudhury, T. R., \& Ratra, B. 2018, MNRAS, 479, 4566 [arXiv:1712.00018] 


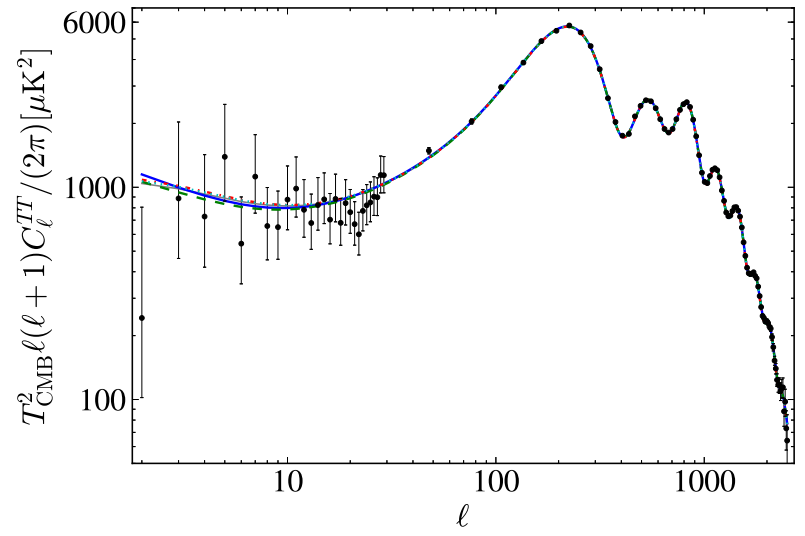

(a)

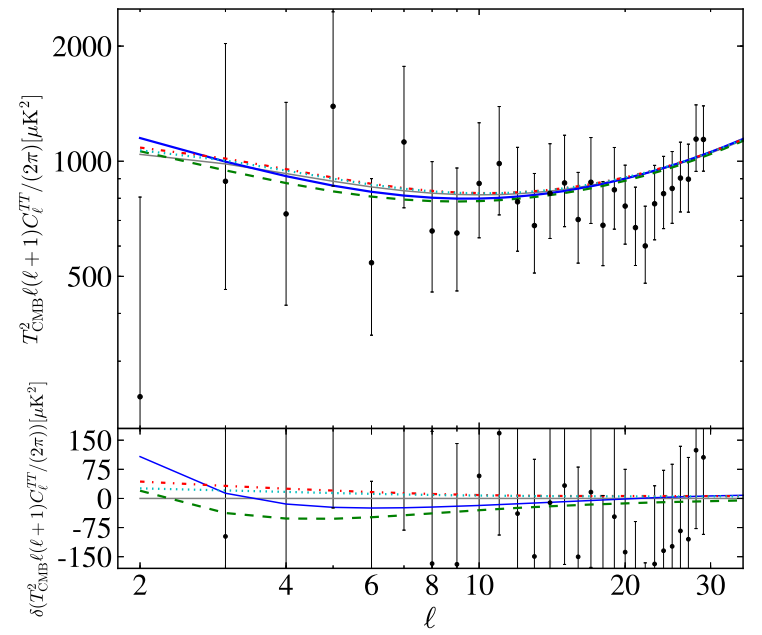

(c)

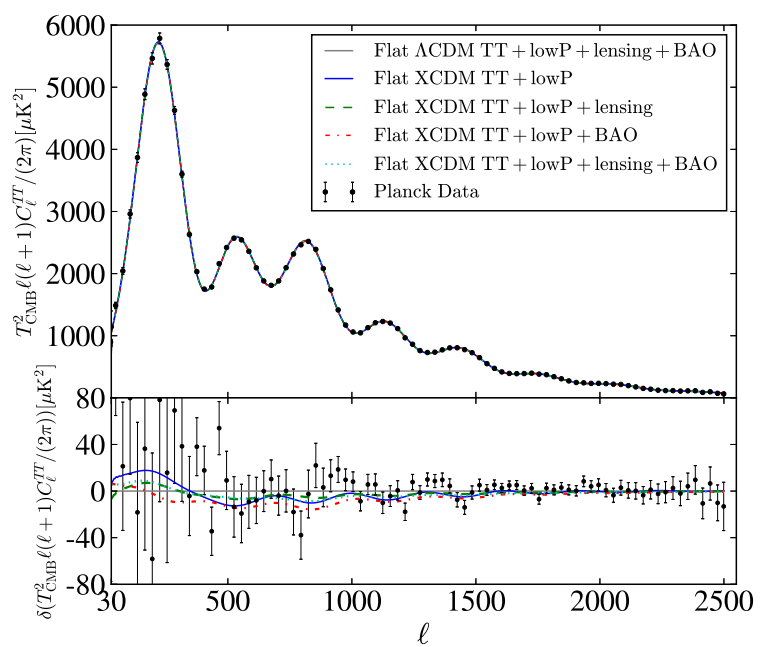

(e)

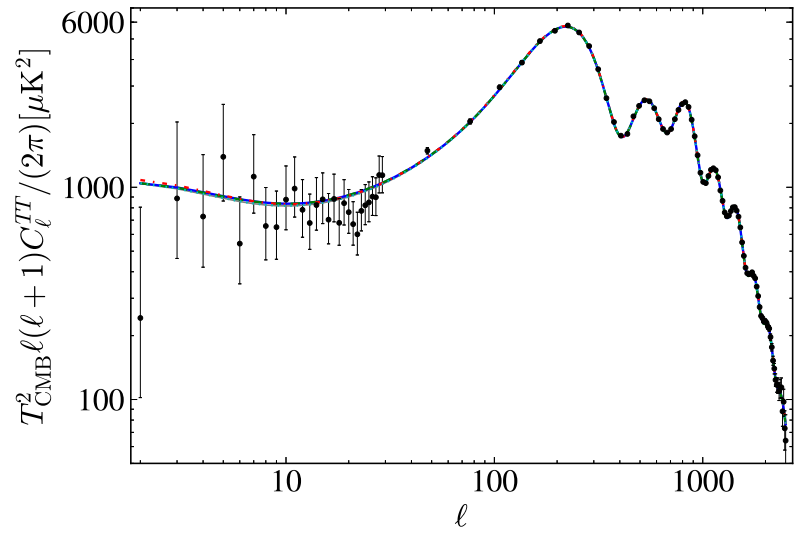

(b)

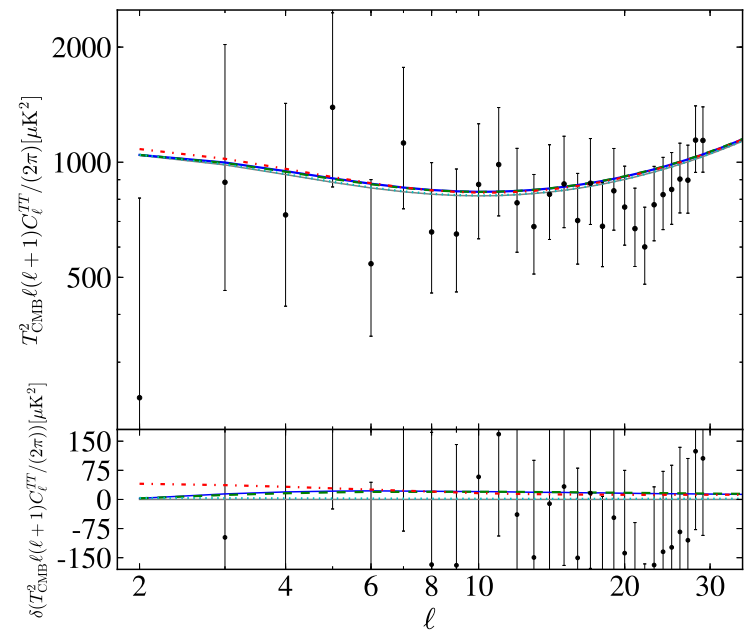

(d)

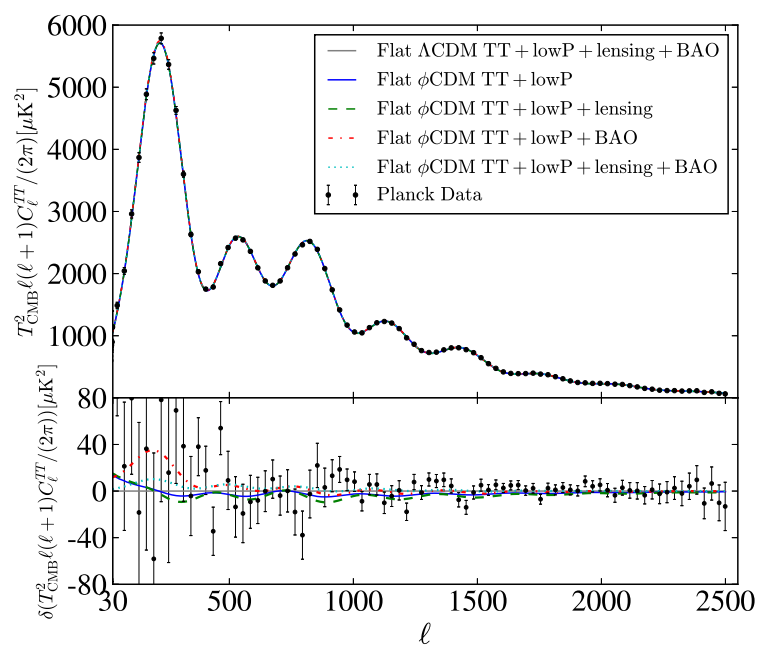

(f)

Figure 3. The best-fit $C_{\ell}$ 's for the XCDM parameterization (left panels (a), (c) and (e)) and the $\phi$ CDM model (right panels (b), (d) and (f)) compared to the spatially-flat tilted $\Lambda$ CDM model (gray solid line). Linestyle information are in the boxes in the two lowest panels. Planck 2015 data are shown as black points with error bars. The top panels show the all- $\ell$ region. The middle panels show the low- $\ell$ region $C_{\ell}$ and residuals. The bottom panels show the high- $\ell$ region $C_{\ell}$ and residuals. 

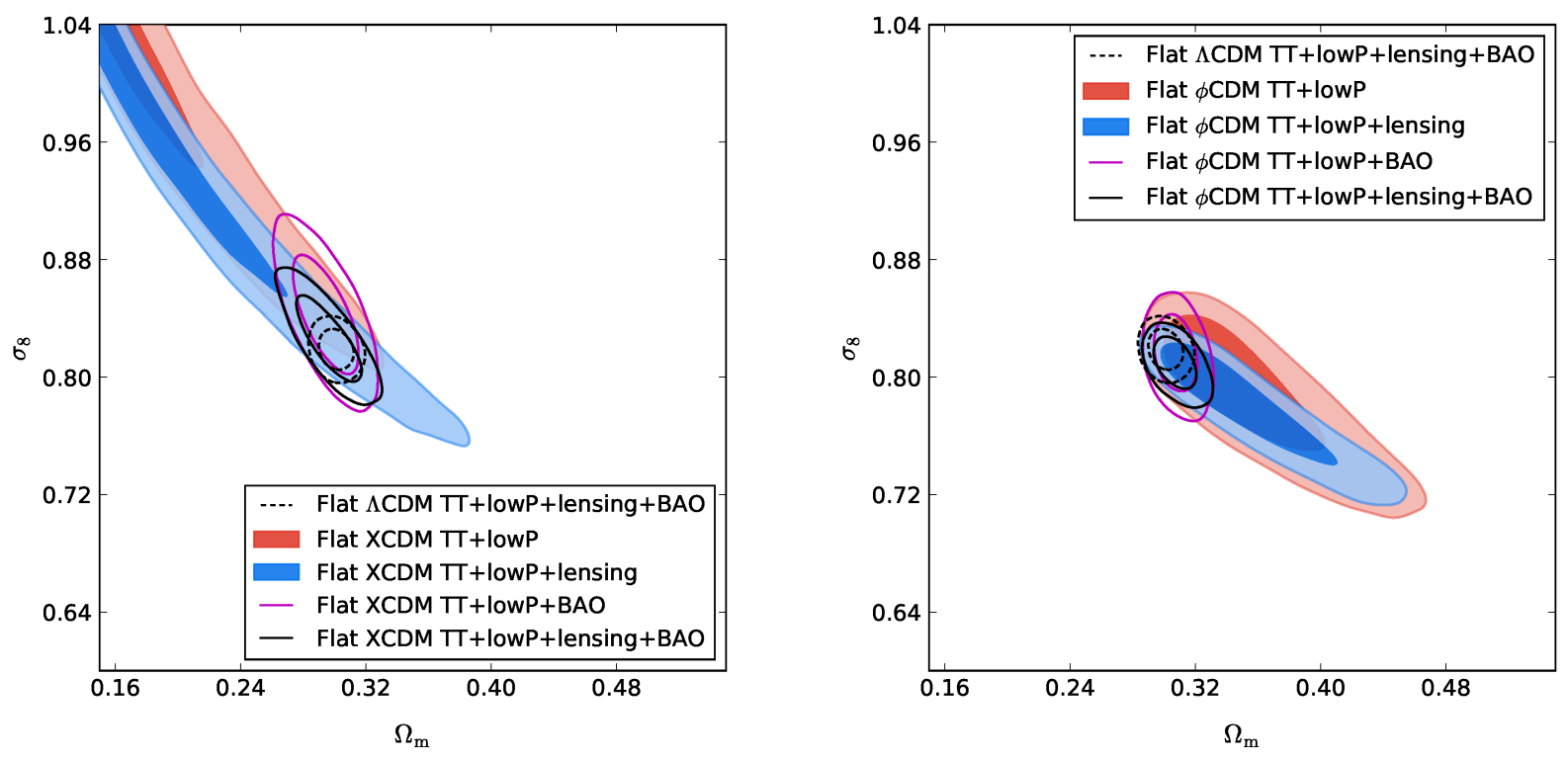

Figure 4. $68.27 \%$ and $95.45 \%$ confidence level contours in the $\sigma_{8}-\Omega_{\mathrm{m}}$ plane.

Mitra, S., Park, C.-G., Choudhury, T. R., \& Ratra, B. 2019, MNRAS, 487, 5118 [arXiv:1901.09927]

Mukherjee, P., Banday, A. J., Riazuelo, A., Górski, K. M., \& Ratra, B. 2003, ApJ, 598, 767 [arXiv:astro-ph/0306147]

Ooba, J., Ratra, B., \& Sugiyama, N. 2018a, ApJ, 864, 80 [arXiv:1707.03452]

Ooba, J., Ratra, B., \& Sugiyama, N. 2018b, ApJ, 869, 34 [arXiv:1710.03271]

Ooba, J., Ratra, B., \& Sugiyama, N. 2018c, ApJ, 866, 68 [arXiv:1712.08617]

Park, C.-G., \& Ratra, B. 2019a, ApJ, 882, 158 [arXiv:1801.00213]

Park, C.-G., \& Ratra, B. 2019b, Ap\&SS, 364, 82 [arXiv:1803.05522]

Park, C.-G., \& Ratra, B. 2018, ApJ, 868, 83 [arXiv:1807.07421]

Park, C.-G., \& Ratra, B. 2019c, Ap\&SS, 364, 134 [arXiv:1809.03598]

Park, C.-G., \& Ratra, B. 2019d, arXiv:1908.08477

Pavlov, A., Farooq, M., \& Ratra, B. 2014, PhRvD, 90, 023006 [arXiv:1312.5285]

Pavlov, A., Westmoreland, S., Saaidi, K., \& Ratra, B. 2013, PhRvD, 88, 123513 [arXiv:1307.7399]

Peebles, P. J. E. 1984, ApJ, 284, 439

Peebles, P. J. E., \& Ratra, B. 1988, ApJ, 325, L17

Penton, J., Peyton, J., Zahoor, A., \& Ratra, B. 2018, PASP, 130, 114001 [arXiv:1808.01490]

Planck Collaboration, Ade, P. A. R., Aghanim, N., Arnaud, M., et al. 2016, A\&A, 594, A13 [arXiv:1502.01589]

Podariu, S., \& Ratra, B. 2001, ApJ, 532, 109 [arXiv:astro-ph/9910527]
Ratra, B. 1985, PhRvD, 31, 1931

Ratra, B. 1989, PhRvD, 40, 3939

Ratra, B. 1992, PhRvD, 45, 1913

Ratra, B. 2017, PhRvD, 96, 103534 [arXiv:1707.03439]

Ratra, B., \& Peebles, P. J. E. 1988, PhRvD, 37, 3406

Ratra, B., \& Peebles, P. J. E. 1995, PhRvD, 52, 1837

Ratra, B., \& Vogeley, M. 2008, PASP, 120, 235 [arXiv:0706.1565]

Riess, A. G., Casertano, S., Yuan, W., et al. 2018, arXiv:1801.01120

Ross, A. J., Samushia, L., Howlett, C., et al. 2015, MNRAS, 449, 835 [arXiv:1409.3242]

Ryan, J., Chen, Y., \& Ratra, B. 2019, MNRAS, 488, 3844 [arXiv:1902.03196]

Ryan, J., Doshi, S., \& Ratra, B. 2018, MNRAS, 480, 759 [arXiv:1805.06408]

Sahni, V., Shafieloo, A., \& Starobinsky, A. A. 2014, ApJ, 793, L4 [arXiv:1406.2209]

Samushia, L., Chen, G., \& Ratra, B. 2007, arXiv:0706.1963

Samushia, L., \& Ratra, B. 2010, ApJ, 714, 1347 [arXiv:0905.3836]

Sievers, J. L., Hlozek, R. A., Nolta, M. R., et al. 2013, JCAP, 1310, 060 [arXiv:1301.0824]

Solà, J., de Cruz Pérez, J., \& Gómez-Valent, A. 2018, Europhys. Lett., 121, 39001 [arXiv:1606.00450]

Solà, J., de Cruz Pérez, J \& Gómez-Valent, A. 2017c, arXiv:1703.08218

Solà, J., Gómez-Valent, A., \& de Cruz Pérez, J. 2015, ApJ, 811, L14 [arXiv:1506.05793]

Solà, J., Gómez-Valent, A., \& de Cruz Pérez, J. 2017a, ApJ, 836, 43 [arXiv:1602.02103] 
Solà, J., Gómez-Valent, A., \& de Cruz Pérez, J. 2017b, Mod. Phys. Lett. A, 32, 1750054 [arXiv:1610.08965]

Solà, J., Gómez-Valent, A., \& de Cruz Pérez, J. 2017d, Phys. Lett. B, 774, 317 [arXiv:1705.06723]

Yashar, M., Bozek, B., Abrahamse, A., Albrecht, A., \& Barnard, M. 2009, PhRvD, 79, 103004 [arXiv:0811.2253]

Yu, H., Ratra, B., \& Wang, F.-Y. 2018, ApJ, 856, 3 [arXiv:1711.03437]
Zhai, Z., Blanton, M., Slosar, A., \& Tinker, J. 2017, ApJ, 850, 183 [arXiv:1705.10031]

Zhang, Y.-C., Zhang, H.-Y., Wang, D.-D., et al. 2017, Res. Astron. Astrophys., 17, 6 [arXiv:1703.08293]

Zhao, G.-B., Raveri, M., Pogosian, L., et al. 2017, Nat. Astron., 1, 627 [arXiv:1701.08165]

Zheng, X., Ding, X., Biesiada, M., Cao, S., \& Zhu, Z.-H. 2016, ApJ, 825, 17 [arXiv:1604.07910] 